\title{
A Novel Methodology to Assess theRelaxation Rate of the Intervertebral Disc by Increments on Intradiscal Pressure
}

\author{
Ângelo R. Araújo ${ }^{a}$, Nuno Peixinho ${ }^{b}$, A. C. Marques Pinho ${ }^{c}$ and J. C. P. Claro ${ }^{d}$ \\ Universidade do Minho, Dep. Mech. Eng.,Campus of Azurém, University of Minho, \\ 4804-533Guimarães, Portugal \\ aangelo14araujo@gmail.com; ${ }^{b}$ peixinho@dem.uminho.pt; cacmpinho@dem.uminho.pt; \\ jicpclaro@dem.uminho.pt
}

Keywords: intervertebral disc; intradiscal pressure.

\begin{abstract}
The Intervertebral Disc (IVD) is subjected to several types of loading during daily routine events. However, the overloading on this structure induces higher Intradiscal Pressure (IDP), which could cause severe damage on its structure. This study describes a new approach to that allows monitorize and pressurize nuclear region of the IVD, with a cartilaginous endplate access, by the insertion of an external fluid, while a Motion Segment (MS-assembly composed by vertebra-disc-vertebra) is compressed at a physiological load. This methodology includes the use of a pneumatic structure that applies a certain pressure on the hydrostatic system, forcing a fluid to enter into the MS through a screw, with a drilled hollow along its entire length. Preliminary results indicated that this methodology presents high potential to efficiently pressurize the IVD, providing a useful tool to better understand the response of this structure under pressure.
\end{abstract}

\section{Introduction}

The hydrostatic pressure on the Nucleus Pulposus (NP), usually known as intradiscal pressure (IDP), varies with the loads applied on intervertebral disc (IVD) during the normal daily activities [1]. Some movements such as compression combined with hyperflexion could generate an IDP beyond what disc could withstand, promoting several injuries [1], [2]. Thus, the study of IDP is a subject of deep interest in order to determine its contribution for the IVD injury.

The methodology used experimentally determine the IDP is typically sensors based[3-10]. These sensors present a great accuracy on the recordings, helping to generate valuable data for a pre-clinically evaluation of spinal implants and disc injuries in further biomechanical in vitro experiments. However, these approaches present some drawbacks such the spinal fluid leaks and the use of annular access, causing irreversible damage on the annular fibers [11]. The monitorization of IDP could provide a better understanding of the mechanisms of IVD failure and treatments. Thus, it is desirable to develop a simple technique that not only monitorizes IDP without Annulus Fibrosus (AF) injury but also presents the capacity to test the variation of IDPs during a compression test.

The objective of the present work is to design a new method that allows monitorize and pressurize nuclear region of the IVD through cartilaginous endplate access. A new combined hydraulic and pneumatic apparatus was designed to study IVD pressurization effect onload relaxation.

\section{Materials and Methods}

Motion segment (MS) collection and preservation.After collected, the lumbar spines from young porcine were immediately sawed into MS[12], which consists on an IVD and its adjoining vertebral bones. Later, some MSs were submitted to test. The remaining samples were sealed in plastic bags and frozen at $-20^{\circ} \mathrm{C}$, until the day prior to mechanical testing, minimizing the tissue dehydration. This procedure was adopted since dead and frozen storage presents a negligible effect on mechanical properties of the spine[13].Before starting any mechanical test, samples were hydrated with $12 \mathrm{~h}$ of PBS (1X).

The pressurization apparatus. The schematic representation of the apparatus is presented in Fig.1. The novel apparatus is divided in two parts: a pneumatic and a hydraulic one. The pressurizing mechanism can be described as a pneumatic structure that applies a certain pressure on the hydrostatic 
system, forcing the fluid to flow into the lumbar MS.On the pneumatic segment, the pressure is exerted by the application of external pressurized air source which acts on a pneumatic double-acting mini-cylinder. The pressure is controlled at the entrance of pneumatic cylinder by a balancing valve. Then, the inner stem of mini-cylinder pushes the internal piston of a stainless steel hydraulic cylinder, specially designed on the scope of this application. The pressure, applied on the hydraulic cylinder, is transmitted to the fluid poured on hydraulic system. The inflow is promoted by the opening of the tap. When it is closed, the hydraulic system is entirely filled with fluid. During this process, care is taken to prevent air bobbles formation. When the tap is closed, the MS is ready to be pressurized. The pressure on the hydraulic segment is monitored immediately upward the fluid insertion on IVD by a manometer. Finally, the fluid is injected on IVD through the perforation on the top of the motion segment.

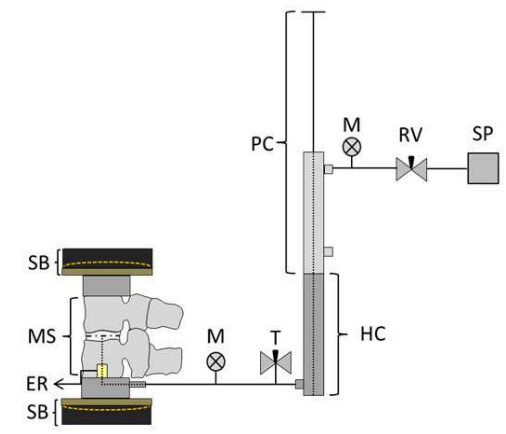

Fig. 1 Schematic representation of pressure apparatus: SP - Pressurized air source; RV - Balancing valve; M-Manometer; PC - Pneumatic cylinder; HC - Hydraulic cylinder; T - Tap; SB - Spherical axial bearing; ER - Epoxy resin; MS - Motion Segment.

Motion segment attachment. The vertebral body presents a viscous structure, being extremely hard to tight a screw on it. To effectively overcome this obstacle, a $9 \mathrm{~mm}$ diameter and $1 / 2$ of top vertebra height hole was drilled on the MS, in order to fill it with an epoxy resin with fast curing. Consequently, a $4 \mathrm{~mm}$ pilot hole was carefully drilled longitudinally through the resin and vertebrae until a sudden change on structure resistance occurs. This change of resistance indicates the point of contact between the endplate and the NP.The MS was attached to pressure apparatus by a self-tapping steel screw (Fig. 2-B).

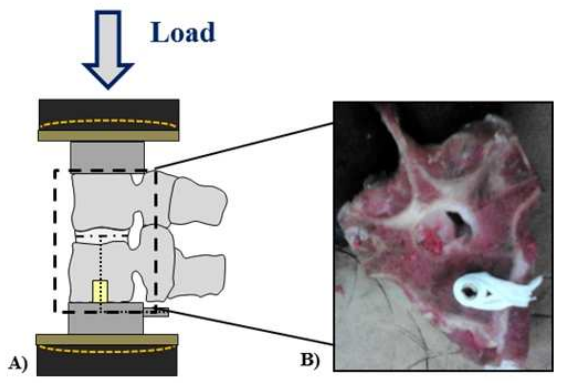

Fig. 2The MS attachment. A) TheMS is placed between two plates on Instron ${ }^{\circledR} 8872$, being subjected to compression. The bottom plate is drilled, allowing the fluid passage from pressure apparatus to the hollowed screw. The attachment is done on epoxy resin (yellow region), to provide a better adhesion of the screw B) Scale-up of the drilled MS.

The self-tapping screw length was $20 \mathrm{~mm}$, presenting two threads: a section of $10 \mathrm{~mm} \times \varnothing 7 \mathrm{~mm}$ drywall screw thread to drill on vertebra and a region of $10 \mathrm{~mm} \times \varnothing 5 \mathrm{~mm}$ to attach to the stainless steel top plate. This screw also presents a drilled hollow along its entire length, with an internal bore of $\varnothing 1.5 \mathrm{~mm}$, allowing the fluid passage.

The screw was tightened until reaching the contact point between cartilaginous endplate and the IVD. In addition, an O-Ring was placed on the stainless steel top plate (around the self-tapping screw) in order to prevent fluid leakage. 
Experimental procedure. Before specimens were loaded, the samples $(n=5)$ were aligned using a transversal dashed line in middle plan of the IVD, as it is visible on Fig. 2. In addition, all samples were positioned at the same place during the test.Once placed on the Instron ${ }^{\circledR} 8872$, the assemble was subjected to a pre load of $50 \mathrm{~N}$, during $5 \mathrm{~min}$, to ensure the contact with loading platen and to minimize errors due to post-mortem effects[12]. Then, a displacement of $2 \mathrm{~mm}$ was imposed to each lumbar porcine MS ( $\mathrm{n}=5)$ during 30 minutes. During this period, the IDP suffered increments of $0.1 \mathrm{MPa}$ each 5 min loading, as presented on Fig. 3.

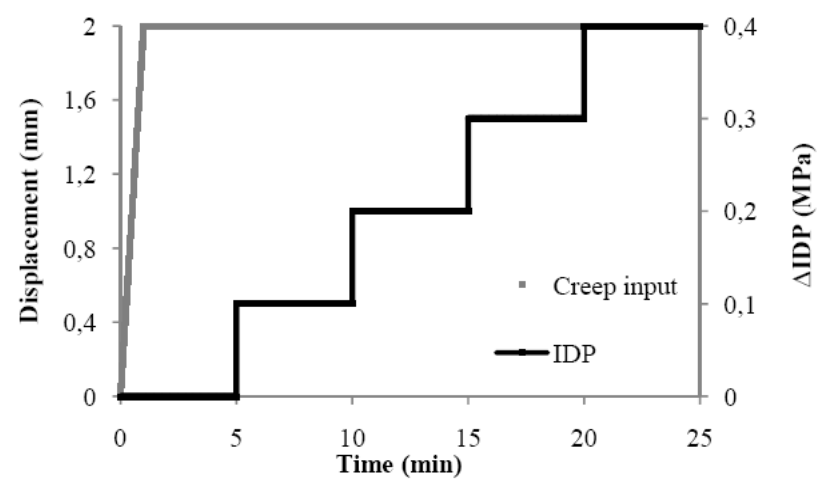

Fig. 3 Mechanical loading protocol used to assess the behavior of IVD under a set IDP. The input data consist of a $2 \mathrm{~mm}$ displacement on each IVD. After periods of $5 \mathrm{~min}$, the pressure increases in

$0.1 \mathrm{MPa}$. The results were registered as load relaxation as function of time.

The pressure was monitored on manometer and the load values were recorded using the Instron ${ }^{\circledR}$ 8872 software BlueHill ${ }^{\circledR}$. After testing, each MS was sawed transversally on the IVD region, in order to assess if the IVD was pressurized on NP region. The goal is to observe if the equipment is able to effectively pressurize the IVD on the required region.

\section{Experimental Results}

Visual inspection of pressurized MS. The tests were performed to assess the functionality of pressurizing system and, in addition, to validate its results. All specimens were pressurized in NP region.All samples showed no degeneration. The specimens that not detected a signal of NP pressurizing were discarded. The average IVD area and height were $917.2 \pm 107.0 \mathrm{~mm}^{2}$ and $5.97 \pm$ $0.63 \mathrm{~mm}^{2}$, respectively.

The effect of $\triangle$ IDP on the relaxation load. An example of the output of the mechanical test performed during this work is presented on Fig.4. This curve clearly shows the effect of the $\Delta$ IDP on the relaxation load: at each IDP increment, it is noticeable a slight load peak. Furthermore, the peak increases with IDP change - for instance, the peak is higher for a 0.2-0.3 MPa change than from $0.1-0.2 \mathrm{MPa}$ increment. This characteristic is common in all performed tests performed, indicating the efficiency of the developed method. It is also visible that the noise around each IDP increment is minimal or almost zero.

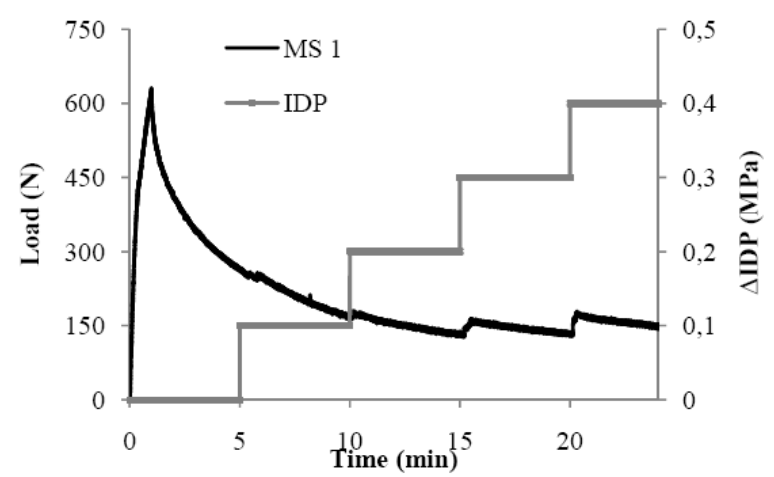

Fig. 4Load-time curves: the dark line represents the relaxation curve for a representative sample (MS

1). The stepped line represents time duration of each IDP increment (with steps of $0.1 \mathrm{MPa}$ ). In addition, it is visible a slight peak of load at each IDP change. 


\section{Conclusions}

This study presents a new methodology for the evaluation of the mechanical response of IVD, under IDP increments, at an imposed compressive strain. Previous studies [3-10] explored the IDP by AF access, causing an irreversible deformation on IVD structure. This pressurization technique uses the endplate access allowing the quantification of mechanical IVD properties without disrupting the AF fibres.

Concerning to the obtained results, it is clear that this approach was able to effectively increment the IDP, with a disposable or null pressure variation during after each increment. In addition, the pressure acted directly on NP meaning that the insertion method is valid.

Analysing Fig. 4, it is visible a load peak after each increment of IDP. These load peaks are likely related to instantaneous augmentation of IDP, which promotes a volumetric omnidirectional disc expansion and consequently generates an increase on load measured on load cell. In addition, the magnitude of load peaks increases with testing time. Thus, the last IDP increment presents the highest load peak. This is expected fact: the load is relaxing reaching minimum values during the last IDP increments. Thus a higher load increment was visible for the same change on IDP.

The data presented generated valid results in order to assess to capacity of the equipment to effectively pressurize the IVD (visible by the peaks on load generate by IDP increment). Moreover, they did not jeopardize the conclusions in terms of load relaxation, since the IDP increment for each step of pressurization is among the physiological range of IDP.

The presented results, provided by this new methodology that encloses an alternative IVD access with the absence of AF disruption, could be important to both understand and compare the influence of the IDP's increment on the response of IVD to the application of external loads. Moreover, the possibility of introducing fluids with higher viscosities than water (with a simple apparatus adaptation), could be useful for analyse the effect of the insertion of different materials, such as hydrogel based NP substitutes.

This new pressure apparatus has still the potential to provide new insights about the mechanisms of IVD failure, namely about the values about what is the pressure it support, and, if associated with a microstructural analysis, to assess the information about possible critical points on annulus fibrosus. Finally, it can be also used to experimentally validate constitutive models of IVD, by the study of the behavior of IVD under the IDP variation.

\section{Acknowledgments}

This work was funded by the project "NP Mimetic - Biomimetic Nano-Fibre Based Nucleus Pulposus Regeneration for the Treatment off Degenerative Disc Disease", financed by the European Commission under FP7 (grant NMP-2009-SMALL-3-CP-FP 246351).

The authors also express their gratitude to Indústria de Carnes do Minho (ICM) - Primor Groupfor the possibility of collecting spine column samples on their facilities.

\section{References}

[1] H. Schechtman, P. A. Robertson, and N. D. Broom: J. Biomech. Vol. 39, no. 8 (2006), pp. 1401-1409.

[2] A. Nachemson and G. Elfström: Scand. J. Rehabil. Med. Suppl. Vol. 1 (1970), pp. 1-40.

[3] A. Nachemson: ActaOrthop. Scand. Vol. 35, (1965), pp. 314-28.

[4] H. Okushima: Nihon GekaHokan, Vol. 39, no. 1 (1970), pp. 45-57.

[5] A. J. Lisi, C. W. O’Neill, D. P. Lindsey, R. Cooperstein, E. Cooperstein, and J. F. Zucherman: J. Appl. Biomech., vol. 22, no. 3 (2006), pp. 234-9.

[6] H. J. Wilke, P. Neef, M. Caimi, T. Hoogland, and L. E. Claes: Spine, vol. 24 (8) (1999), pp. $755-62$. 
[7] K. Sato, S. Kikuchi, and T. Yonezawa: Spine (Phila. Pa. 1976), vol. 24(23) (1999), pp. $2468-74$.

[8] P. a Cripton, G. a Dumas, and L. P. Nolte: J. Biomech., vol. 34, no. 4 (2001), pp. 545-9.

[9] J. Pospiech, D. Stolke, H. J. Wilke, and L. E. Claes: Neurosurgery, vol. 44(2), 1999, pp. 379-385.

[10]C. R. Dennison, P. M. Wild, P. W. G. Byrnes, A. Saari, E. Itshayek, D. C. Wilson, Q. A. Zhu, M. F. S. Dvorak, P. A. Cripton, and D. R. Wilson: J. Biomech., vol. 41, no. 1 (2008), pp. 221-5.

[11]M. K. Moore, S. Fulop, M. Tabib-Azar, and D. J. Hart: Spine J., vol. 9, no. 12 (2009), pp. $1030-4$.

[12]N. H. Campbell-Kyureghyan, S. V. Yalla, M. Voor, and D. Burnett: J. Mech. Behav. Biomed.Mater., vol. 4, no. 4 (2011), pp. 549-557.

[13]M. A. Adams, D. S. McNally, and P. Dolan: J. Bone Joint Surg. Br., vol. 78, no. 6 (1996), pp. 965-72. 
Mechatronics and Mechanical Engineering I

10.4028/www.scientific.net/AMM.664

\begin{abstract}
A Novel Methodology to Assess the Relaxation Rate of the Intervertebral Disc by Increments on Intradiscal Pressure
\end{abstract}

10.4028/www.scientific.net/AMM.664.379 